\title{
Chocolate Terahertz Fresnel Lens
}

\author{
Mateusz Surma, ${ }^{* 1}$ Paweł Komorowski, ${ }^{2}$ Maciej Neneman, ${ }^{1}$ and Agnieszka Siemion ${ }^{1}$ \\ ${ }^{1}$ Faculty of Physics, Warsaw University of Technology, Koszykowa 75, 00-662 Warsaw, Poland \\ ${ }^{2}$ Institute of Microelectronics and Optoelectronics, Warsaw University of Technology, Koszykowa 75, 00-662 \\ Warsaw, Poland
}

Received September 16, 2020; accepted December 09, 2020; published December 17, 2020

\begin{abstract}
Recent enorSSmous development of 3D printing techniques gave the possibility of precise manufacturing of designed optical structures. This paper presents designing, manufacturing and the results obtained for a chocolate Fresnel lens. Chocolate, similarly to wax, can be melted and used in the 3D printed form to create a terahertz (THz) optical element. The parameters of a chocolate lens are compared with those of one made of wax. In simple applications both materials can be used as a cost-effective alternative for conventional optical materials used for a $\mathrm{THz}$ range of radiation. Both lenses have been designed and compared for $140 \mathrm{GHz}$.
\end{abstract}

Transmissive terahertz (THz) optics is typically made from polymers, such as PTFE, HDPE or TPX, which in most cases require a milling process in manufacturing optical elements [1]. The need for cheap alternatives of conventional terahertz materials has already been noticed [2-4]. The growing precision and diversity of used materials in 3D printing techniques makes this method a competitive solution in the case of designing $\mathrm{THz}$ optical elements (especially more detailed - diffractive ones). Moreover, the 3D printing method can also be used to fabricate molds that can be filled with the desired material to form a $\mathrm{THz}$ diffractive optical element (DOE). We focused on substances that are easy to melt and process allowing fast and easy manufacturing of diffractive components. Chocolate has been shown to be transparent in a $\mathrm{THz}$ band when tested for foreign bodies [5]. The biggest advantage of chocolate is that it can be processed with simple and inexpensive equipment. It has been shown that $3 \mathrm{D}$ printing can be used to produce $\mathrm{THz}$ optical structures [4], also diffractive [6] and having complex shapes [7-12]. However, using a 3D printing method is mostly limited to dielectric materials like polymers, since many other 3D printable materials are not transparent enough for $\mathrm{THz}$ radiation (e.g., polylactic acid - PLA), which may be disadvantageous. Thus, the novelty is using chocolate as an example of brittle material for $\mathrm{THz}$ optics and silicone molding procedure allowing for safe extraction of such a brittle element. There are three methods of mold production described and compared.

Firstly, different brands and kinds of chocolate were formed into flat discs to measure optical properties of

${ }^{*}$ E-mail: mateusz.surma.dokt@pw.edu.pl materials. The results of absorption coefficient and refractive index measurements are presented in Fig 1. A characteristic local absorption peak can be clearly seen above $0.5 \mathrm{THz}$ for white and milk chocolates. The analysis of the ingredients of different chocolates suggests that this peak is related to the presence of lactose [12] in white and milk chocolates. It is worth noticing that for the frequency of the absorption peak the drop of a refractive index value can also be observed [13]. Based on these data, melting properties (depending mostly on the amount of cocoa fat) and strength of the manufactured lens dark chocolate (Wedel) was chosen for final lens fabrication.

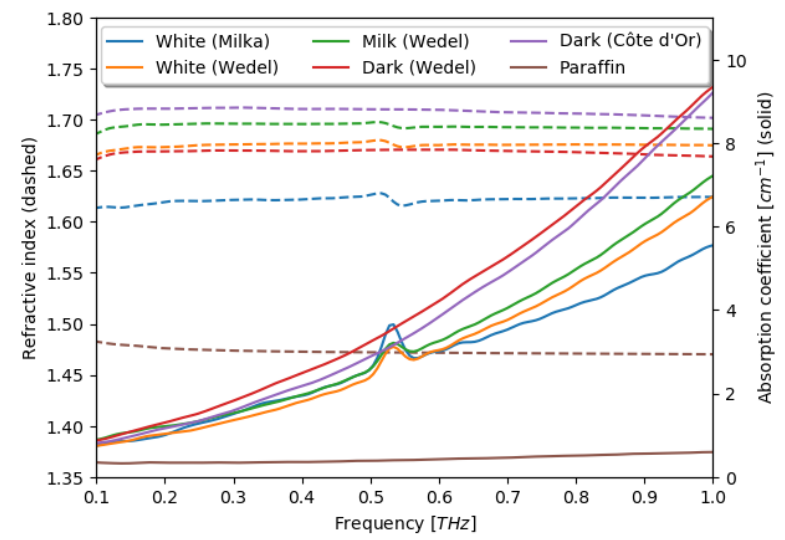

Fig. 1. The refractive index (dashed) and the absorption coefficient (solid) of used materials: five types of chocolate (two white, two dark and one milk) and paraffin for comparison.

A Fresnel kinoform diffractive lens was designed for $140 \mathrm{GHz}$ (corresponding to $2.14 \mathrm{~mm}$ wavelength) using Light Sword 6.0 software. The phase transmittance of the lens was calculated with the equation describing a nonparaxial approach (1) in which $k$ stands for the wavenumber, $r$ for the radius in the lens plane and $f$ for its focal length.

$$
\theta=-k \sqrt{r^{2}+f^{2}}
$$

To calculate the proper thickness of the structure the design wavelength of $\mathrm{THz}$ radiation and refractive index of chocolate must be taken into account $-n_{c h}=1.67$ for the $2.14 \mathrm{~mm}$ wavelength $(140 \mathrm{GHz})$. This value 
corresponds to relatively low Fresnel reflection losses of approx. 8\%. The absorption coefficient of selected material for $140 \mathrm{GHz}$ reached $1.05 \mathrm{~cm}^{-1}$. The focal length and the diameter were set to $100 \mathrm{~mm}$. The phase distribution (which is the representation of the introduced lens's phase delay map) was exported to a BMP file (Fig. 2.A) for further processing.

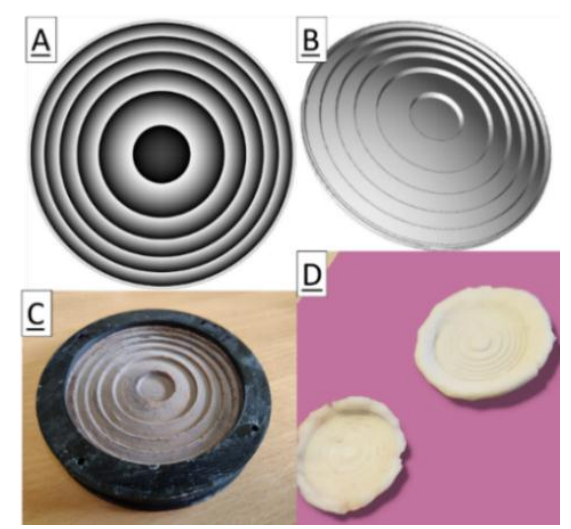

Fig. 2. A picture of: (A) bmp file with the kinoform phase distribution (white corresponds to $2 \Pi$ phase change and black to 0п), (В) stl file of lens based on the designed phase distribution, $(C)$ chocolate lens in $3 D$ printed holder and (D) used molds.

3D printing was used at the first attempt to produce the mold. A negative of the lens phase distribution was converted to a 3D STL model (forming concave mold). The depth of the modeled mold was calculated as the height of the kinoform of first order $h$ described with the equation (2) and was evaluated to be equal to $3.19 \mathrm{~mm}$. The substrate of a kinoform was set to approx. $1 \mathrm{~mm}$ summing up for a total lens height of $4.19 \mathrm{~mm}$.

$$
h=\frac{\lambda}{\left(n_{c h}-1\right)}
$$

The mold was printed using an additive printer Original Prusa i3 MK3S (with a layer height of $0.1 \mathrm{~mm}$ and a nozzle size of $0.4 \mathrm{SSmm}$ ) and then used to shape the melted chocolate. The extraction of kinoforms from 3D printed molds was difficult and often caused deformation and loss of many details in diffraction zone parts of the lens due to the brittleness of the lens and the lack of flexibility of the mold. To overcome the difficulties with extraction, a positive mold was 3D printed (visualization in Fig. 2.B) to transfer the desired shape into a more flexible mold material - silicone. The dimensions of the positive mold were set according to the dimensions mentioned before.

Two methods of manufacturing silicone molds were tested. In the first method the silicone was placed in a warm water bath and later shaped by pressing the $3 \mathrm{D}$ printed form (with the same printing parameters) into it. Small amount of liquid soap was used on silicone to reduce sticking of the mold to the surface. This method produces very flexible but also fragile molds.
Another tested method consisted in mixing silicone with cornstarch. Created molds were much sturdier while maintaining sufficient flexibility for extracting chocolate kinoforms. Both molds are depicted in Fig. 2.D, together with the finally manufactured chocolate $\mathrm{THz}$ diffractive lens in Fig. 2.C.

The 3D printing method for mold construction described above was earlier employed for the production of a wax lens. The focal length and diameter of the lens were analogous to those of the chocolate lens.

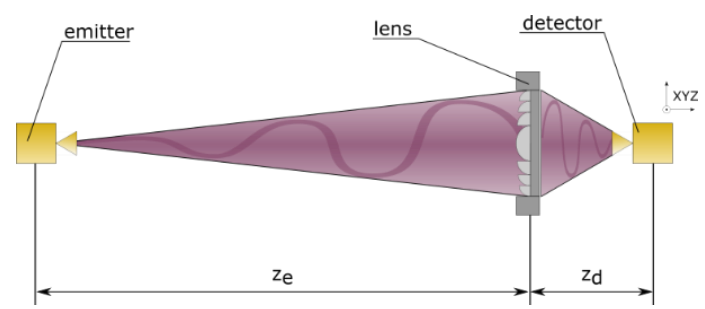

Fig. 3. The scheme of measurement setup.

To test the optical properties of prepared lenses, a simple evaluation setup was developed (Fig. 3). It consisted of a $\mathrm{THz}$ emitter followed by a tested lens placed $\mathrm{z}_{\mathrm{e}}=1 \mathrm{~m}$ after an emitter and detector in the focal place of a lens $\left(\mathrm{z}_{\mathrm{d}} \approx 11 \mathrm{~cm}\right.$ behind lens - calculated with the thin lens formula). Frequency multiplier chains (by Virginia Diodes Inc. - VDI) were used as a source and a detector of $\mathrm{THz}$ radiation. A detector was placed on motorized stages allowing for scanning measurements of focal spots in three directions.

The focal spots for both chocolate and wax lenses have been scanned in the Fourier plane (Fig. 4) and along the axis of propagation (Fig. 5). The measuring step was equal to $2 \mathrm{~mm}$ with the diameter of the horn equal to 8.4 $\mathrm{mm}$. Scans of intensity distributions in Fourier planes are complemented by the intersection through the point with a maximal measured value and are located on the sides of images. The dotted circles represent theoretically calculated Airy disc size. The Airy disc calculated accordingly to the formula (3) [14], where $\lambda$ is the wavelength, $\mathrm{f}$ is the focal length and $\mathrm{d}$ is the diameter of the lens has the diameter of $5.22 \mathrm{~mm}$ in both cases. It must be noted that this assumes full and constant illumination of the aperture of the lens. In the case of nonuniform illumination the effective aperture would be smaller, resulting in higher diameter of the Airy disc. Therefore, both theoretical results of the diameter and full width at half maximum (FWHM) of the Airy disc should be rather treated as a lower limit of these values.

$$
A_{d}=2.44 \frac{\lambda \cdot f}{d}
$$

Knowing that the Airy disc is the spot inside the ring representing the $1^{\text {st }}$ minimum of the Bessel function, one can calculate the FWHM, which is equal to $2.2 \mathrm{~mm}$. Moreover, in Fig. 4 the intersections have FWHMs 
depicted by dashed lines. FWHM of vertical/horizontal intersections for chocolate and wax lenses are 4.01/4.35 $\mathrm{mm}$ and 5.04/4.69 $\mathrm{mm}$, respectively.

It can be noticed that the focal spot obtained from the wax lens is brighter than the one from the chocolate lens (the maximum of the chocolate lens focal spot corresponds to the $60 \%$ of the maximum of the wax lens focal spot). Nevertheless, it is expected, as the absorption coefficient of the chocolate used is higher than the absorption coefficient of the wax. Both focal spots have similar diameters, seemingly more than twice wider than the diffraction limit. However, when estimating the portion of energy within the marked diffraction limit, one obtains $62 \%$ and $73 \%$ for the chocolate and wax lenses, respectively. Thus, in both cases most of the energy is focused within the lower limit of the diffraction limit with Gaussian shaped distributions.

(a) Chocolate lens

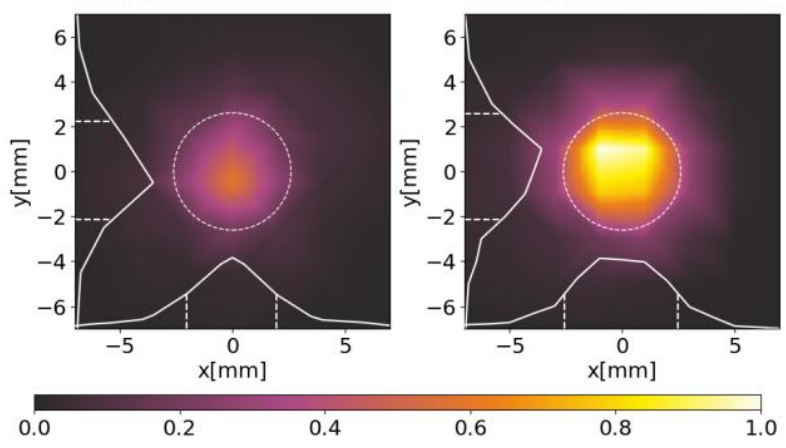

Fig. 4. The scans of the focal spot of the chocolate lens (a) and the reference wax lens (b). The colors of both images are normalized to the highest registered value from both distributions. The dashed circle represents the diffraction limit for both lenses. The left and bottom edges represent the cross-sections through the center of distribution. The dashed lines on cross-sections show the size of a full width at half maximum (FWHM).

The presented techniques allow to produce relatively efficient and cheap $\mathrm{THz}$ optical components. The optical performance of the chocolate lens is not as efficient as the reference wax kinoform, which results from the difference in the absorption coefficient values. However, chocolate is easier to process and removing it from the form is simpler. Thus, it has been shown that various kinds of chocolate can be used as a material for manufacturing $\mathrm{THz}$ optics. One can argue that this material has poor mechanical and thermal properties as well as high risk of spoiling (as an organic, edible substance), which excludes it from practical use. Nevertheless, the goal of these experiments is to show that extremely simple production methods and everyday substances can be successfully used to manufacture working optical elements for a $\mathrm{THz}$ spectral range even from very brittle materials. And even such perishable materials can be used for fast and very cheap prototyping of DOEs.

(a) Chocolate lens

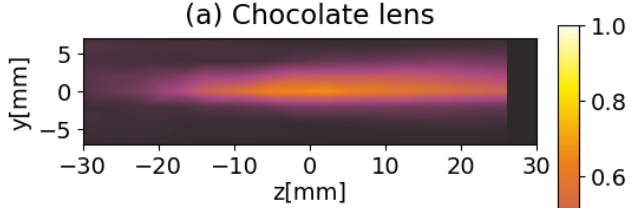

(b) Wax lens

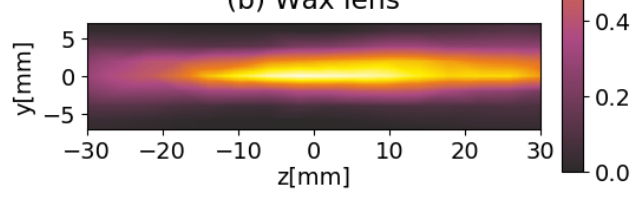

Fig. 5. The visualization of power distribution along the beam produced by chocolate (a) and wax (b) lenses. The colors of both images are normalized to the highest registered value for both.

Since commercially available chocolate comes in many kinds the authors were unable to test the terahertz optical properties of all different types. An interesting subject for further research might be characterizing the optical properties of chocolate in relation to its type and ingredients. Up to now, it can be observed that big differences were observed between dark and white/milk chocolate, however, differences are also present in the case of similar types of chocolate from different manufacturers.

\section{References}

[1] M. Naftaly, R.E. Miles, P.J. Greenslade, Joint 32nd International Conference on Infrared and Millimeter Waves and the 15th International Conference on Terahertz Electronics, 819-820 (2007).

[2] S. Firoozabadi, F. Beltran-Mejia, A. Soltani, D. Jahn, S.F. Busch, J.C. Balzer, M. Koch, 42nd International Conference on Infrared, Millimeter, and Terahertz Waves (IRMMW-THz), 1-2 (2017).

[3] D. Headland, W. Withayachumnankul, M. Webb, H. EbendorffHeidepriem, A. Luiten, D. Abbott, Opt. Expr. 24(15), 17384 (2016).

[4] S.F. Busch, M. Weidenbach, M. Fey, F. Schäfer, T. Probst, M. Koch, J. Infrared Millimeter Terahertz Waves 35(12), 993 (2014).

[5] C. Jördens, and M. Koch, Optical Engineering 47(3), 037003 (2008).

[6] A.D. Squires, E. Constable R.A. Lewis, J. Infrared Millimeter Terahertz Waves 36(1), 72 (2015).

[7] W.D. Furlan, V. Ferrando, J.A. Monsoriu, P. Zagrajek, E. Czerwińska, M. Szustakowski, Opt. Lett. 41(8), 1748 (2016).

[8] X. Wei, C. Liu, L. Niu, Z. Zhang, K. Wang, Z. Yang, J. Liu, Appl. Opt. 54(36), 10641 (2015).

[9] S. Banerji, B. Sensale-Rodriguez, Micro-and Nanotechnology Sensors, Systems and Applications XI 10982, 109822X (2019).

[10] M. Surma, I. Ducin, P. Zagrajek, A. Siemion, Appl. Scien. 9(4), 659 (2019).

[11] A. Siemion, P. Komorowski, M. Surma, I. Ducin, P. Sobotka, M. Walczakowski, E. Czerwińska, Opt. Expr. 28(1), 715-723 (2020).

[12] E.R. Brown, J.E. Bjarnason, A.M. Fedor, T.M. Korter, Appl. Phys. Lett. 90(6), 061908 (2007).

[13] M. Bernier, F. Garet, J.L. Coutaz, Terahertz Spectroscopy-Cutting Edge Technology (Intech-Open Science 2017).

[14] E.Hecht, Optics 5th global ed. (Boston, Pearson Education 2017). 